\title{
Renal Interstitial Fibrosis: An Imperfect Predictor of Kidney Disease Progression in Some Patient Cohorts
}

\author{
Hanni Menn-Josephy ${ }^{a} \quad$ Carol S. Lee ${ }^{a} \quad$ Angela Nolin $^{a}$ Marta Christov ${ }^{c}$ \\ Denis V. Rybin $^{d}$ Janice M. Weinberg ${ }^{\mathrm{e}}$ Joel Henderson ${ }^{b}$ Ramon Bonegio $^{a}$ \\ Andrea Havasi ${ }^{\mathrm{a}}$ \\ ${ }^{a}$ Department of Medicine and ${ }^{\mathrm{b}}$ Department of Pathology, Boston University Medical Center, ${ }^{\mathrm{C}}$ Division of \\ Nephrology, Beth Israel Deaconess Medical Center, and Harvard Medical School Boston, and ${ }^{\mathrm{d}}$ Data Coordinating \\ Center and ${ }^{\mathrm{e} D e p a r t m e n t}$ of Biostatistics, Boston University School of Public Health, Boston, Mass., USA
}

\section{Key Words}

Chronic kidney disease · End-stage renal disease ·

Kidney biopsy · Interstitial fibrosis · Prognosis

\begin{abstract}
Background: The extent of interstitial fibrosis on kidney biopsy is regarded as a prognostic indicator and guide to treatment. Patients with extensive fibrosis are assigned to supportive treatments with the expectation that they have advanced beyond the point at which immunosuppressive or other disease-modifying therapies would be of benefit. Our study highlights some of the limitations of using interstitial fibrosis to predict who will develop end-stage renal disease (ESRD). Methods: Analysis of 434 consecutive renal biopsies performed between 2001 and 2012 at a single center. We assessed the influence of various clinical factors along with fibrosis as predictors of ESRD and dialysis-free survival in various patient groups. Results: Interstitial fibrosis performed well overall as a predictor of progression to dialysis. On average, patients with $>50 \%$ fibrosis progressed more rapidly than those with either $25-49$ or $0-24 \%$ fibrosis with a median
\end{abstract}

\section{KARGER}

E-Mail karger@karger.com

www.karger.com/ajn time to dialysis of $1.2,6.5$ and $>10$ years, respectively. In contrast, interstitial fibrosis was of less value as a predictor of disease progression in a subset of cases that included patients over the age of 70 and those with diabetic nephropathy on biopsy. Surprisingly, $13.9 \%$ of patients with normal renal function had $25-49 \%$ fibrosis and $5 \%$ had more than $50 \%$ fibrosis on biopsy, and 5 years after undergoing biopsy $21 \%$ of patients with $>50 \%$ fibrosis still remained dialysis free. Conclusion: Renal fibrosis is an imperfect prognostic indicator for the development of ESRD and caution should be exercised in applying it too rigidly, especially in elderly or diabetic patients.

(c) 2016 S. Karger AG, Basel

\section{Introduction}

Early identification of individuals with renal disease and implementation of renoprotective treatment can slow or prevent progression to end-stage renal disease (ESRD) reducing morbidity, mortality and improving quality of life. Renal biopsies are indicated to determine 
the underlying pathology, guide therapy, and ascertain the degree of active (potentially reversible) and chronic (considered irreversible) changes [1-7]. Chronic renal damaged is characterized by a variety of morphological changes that are common findings in different renal diseases. In the glomerular compartment, the major modifications are mesangial expansion and glomerulosclerosis; in the tubulointerstitial compartment, the characteristic modifications are tubular atrophy, interstitial fibrosis, and a reduction in the number of capillaries $[2,4]$. Although the majority of ESRD patients in the United States have primary or secondary forms of glomerular disease, studies have suggested that it is actually the extent of accompanying histologic injury in the tubulointerstitium that correlates best with renal function decline $[1,5-12]$. Strictly applied, finding advanced fibrosis on renal biopsy might limit the use of beneficial but potentially toxic treatments based on the assumption of futility. In this study, we assessed the influence of other factors along with fibrosis as predictors of progression to dialysis and examined the predictive value of interstitial fibrosis in various patient groups. The amount of interstitial fibrosis was taken directly from the biopsy report. We intentionally did not validate the reported fibrotic index using morphometric techniques because in real life, clinical decisions are based on standard biopsy reports.

\section{Methods}

We performed a retrospective analysis of renal biopsies of adult patients at Boston University Medical Center (BUMC) between January 2001 and July 2012. All patients who underwent a successful kidney biopsy in this period were included in our initial evaluation, but we excluded 6 patients from subsequent analyses who had acute, reversible renal failure. The following data were collected from the patients' electronic medical record: demographic information, body mass index (BMI), urine protein-to-creatinine ratio, and serum creatinine. Glomerular filtration rate (GFR) was estimated using the 4 variable Modification of Diet in Renal Disease equation for patients who were less than 70 years of age [13] and Chronic Kidney Disease (CKD) Epidemiology Collaboration creatinine equation for patients older than 70 years. The presence or absence of comorbidities including hypertension (HTN), insulin-dependent diabetes mellitus (IDDM), non-insulin-dependent diabetes mellitus (DM), coronary artery disease (CAD), stroke, peripheral vascular disease (PVD), and autoimmune (AI) disease were noted. The following diseases were grouped under the AI category: vasculitis, lupus nephritis, immune complex glomerulonephritis, and post-infectious glomerulonephritis. Histological findings were noted from the biopsy report and included the degree of glomerulosclerosis, and the percentage of tubular atrophy and interstitial fibrosis. If there was more than one pathological finding on the biopsy, it was classified according to the most prev- alent finding. Biopsy findings were categorized as having immunoglobulin A nephropathy, AI renal disease, diabetic nephropathy, or 'other nephropathy' (focal segmental glomerulosclerosis, hypertensive glomerulosclerosis, collapsing glomerulopathy, primary membranous nephropathy, thin basement membrane disease, interstitial nephritis, minimal change disease, or amyloidosis).

Serum creatinine at the time of biopsy and last creatinine available during the study period and/or time of dialysis initiation or death were collected.

The 2 primary end points of the study were initiation of dialysis and the time from kidney biopsy to the initiation of dialysis. We also evaluated a composite outcome of death without reaching dialysis or dialysis as a secondary outcome measure. Only 4 patients declined dialysis.

We described study sample characteristics via means and SDs for continuous variables and counts and percentages for categorical variables. Demographic factors, comorbidities, and laboratory measures were assessed as possible predictors of dialysis. We used a t test for continuous variables and Fisher's exact test for categorical variables to assess bivariate associations. Based on the current literature $[14,15]$, we designed prediction models using clinical and biopsy variables to predict progression to dialysis. The following variables were included in the multivariable logistic model predicting the initiation of dialysis: demographic variables including age, sex, race, and BMI; comorbid conditions including IDDM, HTN, and composite of CAD, PVD, or stroke; laboratory variables at the time of the biopsy including GFR, creatinine, and proteinuria; biopsy findings including percentage of fibrosis, glomerulosclerosis, and nephropathy type. A parsimonious model was constructed using a backward elimination procedure with a 0.2 level to stay in the model. The model that includes fibrosis only was then evaluated. The discriminative ability of the models was described using C-statistic and receiver operating characteristic (ROC) curve. The models' fit was assessed using a Hosmer-Lemeshow test. The parsimonious model was compared to the fibrosis-only model using a likelihood ratio test. The effects were expressed via adjusted ORs with corresponding 95\% CIs. The predicted probability of patients reaching dialysis based on the model including fibrosis only was assessed in various subgroups. A t test was used to compare predicted probability in patients who reached dialysis and patients who did not reach dialysis. We confirmed that there was a linear relationship between fibrosis and dialysis in our data (online suppl. table 6; for all online suppl. material, see www.karger.com/doi/10.1159/000449511). The amount of interstitial fibrosis was taken directly from the biopsy report prepared by an experienced, board-certified nephropathologist using standard diagnostic criteria [1]. We intentionally did not validate the reported fibrotic index using morphometric techniques because clinical decisions are based on a standard biopsy report.

Time to the initiation of dialysis was assessed in patients grouped based on the amount of fibrosis. Kaplan-Meier curves and log-rank test were used in unadjusted analyses. The proportional hazard Cox-regression model was used to adjust for potential confounders; these included the same variables as the dialysis prediction model. The effects were expressed via adjusted hazard ratios (HRs) with corresponding 95\% CI. Analysis was performed using SAS 9.3 software (SAS Institute Inc., Cary, N.C., USA) and $p$ value $<0.05$ was defined as statistically significant. 
Table 1. Patient characteristics of the overall cohort and a bivariate analysis comparing the dialysis group with the non-dialysis group

\begin{tabular}{|c|c|c|c|c|}
\hline Characteristic & Overall $(n=434)$ & Dialysis $(\mathrm{n}=136)$ & No dialysis $(\mathrm{n}=298)$ & $\mathrm{p}$ value \\
\hline \multicolumn{5}{|l|}{ Age } \\
\hline Mean \pm SD & $45.1 \pm 16.3$ & $48.7 \pm 16.2$ & $43.4 \pm 16.1$ & \multirow[t]{2}{*}{0.002} \\
\hline Median and range & $44(17-89)$ & $49(20-83)$ & $42(17-89)$ & \\
\hline \multicolumn{5}{|l|}{ Gender } \\
\hline Male & $226(52.1)$ & $86(38.1)$ & $140(61.9)$ & \multirow[t]{2}{*}{0.002} \\
\hline Female & $208(47.9)$ & $50(24)$ & $158(76)$ & \\
\hline \multicolumn{5}{|l|}{ Race } \\
\hline White & $96(22.1)$ & $26(27.1)$ & $70(72.9)$ & \multirow[t]{4}{*}{0.045} \\
\hline Black & $183(42.2)$ & $71(38.8)$ & $112(61.2)$ & \\
\hline Hispanic & $95(21.9)$ & $24(25.3)$ & $71(74.7)$ & \\
\hline Other & $60(13.8)$ & $15(25)$ & $45(75)$ & \\
\hline \multicolumn{5}{|l|}{ BMI, $\mathrm{kg} / \mathrm{m}^{2}$} \\
\hline Mean $\pm S D$ & $28.9 \pm 7$ & $28 \pm 6.7$ & $29.3 \pm 7.1$ & \multirow[t]{2}{*}{0.070} \\
\hline Median and range & $27.2(13.7-58.8)$ & $26.8(17.2-53.8)$ & $27.7(13.7-58.8)$ & \\
\hline \multicolumn{5}{|l|}{$\mathrm{BMI}, \mathrm{kg} / \mathrm{m}^{2}$} \\
\hline Underweight (16-18.5) & $5(1.2)$ & $2(40)$ & $3(60)$ & \multirow[t]{4}{*}{0.143} \\
\hline Normal (18.5-25) & $129(29.9)$ & $50(38.8)$ & $79(61.2)$ & \\
\hline Overweight (25-30) & $144(33.3)$ & $40(27.8)$ & $104(72.2)$ & \\
\hline Obese $(>30)$ & $154(35.6)$ & $43(27.9)$ & $111(72.1)$ & \\
\hline \multicolumn{5}{|l|}{ Nephropathy } \\
\hline $\mathrm{AI}$ & $124(28.6)$ & $29(23.4)$ & $95(76.6)$ & \multirow[t]{4}{*}{$<0.001$} \\
\hline $\operatorname{Ig} \mathrm{A}$ & $58(13.4)$ & $17(29.3)$ & $41(70.7)$ & \\
\hline Diabetic & $42(9.7)$ & $25(59.5)$ & $17(40.5)$ & \\
\hline Other & $210(48.4)$ & $65(31)$ & $145(69)$ & \\
\hline \multicolumn{5}{|l|}{ Interstitial fibrosis, \% } \\
\hline Mean \pm SD & $31.2 \pm 25$ & $51.6 \pm 23.7$ & $21.9 \pm 19.4$ & \multirow[t]{2}{*}{$<0.001$} \\
\hline Median and range & $25(0-100)$ & $50(5-100)$ & $20(0-80)$ & \\
\hline \multicolumn{5}{|l|}{ Glomerulosclerosis, \% } \\
\hline Mean \pm SD & $25 \pm 24.5$ & $40.1 \pm 28.4$ & $18.1 \pm 18.8$ & \multirow[t]{2}{*}{$<0.001$} \\
\hline Median and range & $16.7(0-100)$ & $40.6(0-100)$ & $10.5(0-77.3)$ & \\
\hline \multicolumn{5}{|c|}{ Creatinine at the time of biopsy, $\mathrm{mg} / \mathrm{dl}$} \\
\hline Mean \pm SD & $2.6 \pm 2.6$ & $4.3 \pm 3.2$ & $1.8 \pm 1.9$ & \multirow[t]{2}{*}{$<0.001$} \\
\hline Median and range & $1.7(0.4-20.3)$ & $3.2(0.6-20.3)$ & $1.2(0.4-15.2)$ & \\
\hline \multicolumn{5}{|c|}{ GFR (MDRD) at the time of biopsy } \\
\hline Mean \pm SD & $57.6 \pm 46$ & $29.4 \pm 28.9$ & $70.5 \pm 46.6$ & $<0.001$ \\
\hline Median and range & $42.5(2.5-246.2)$ & $22.2(2.5-153.1)$ & $61.1(3.7-246.2)$ & \\
\hline GFR at the time of biopsy, $n$ & & & & \\
\hline $90+$ & $107(24.7)$ & $8(7.5)$ & $99(92.5)$ & $<0.001$ \\
\hline $60-89$ & $65(15)$ & $9(13.8)$ & $56(86.2)$ & \\
\hline $30-59$ & $105(24.2)$ & $23(21.9)$ & $82(78.1)$ & \\
\hline $15-29$ & $85(19.6)$ & $48(56.5)$ & $37(43.5)$ & \\
\hline$<15$ & $72(16.6)$ & $48(66.7)$ & $24(33.3)$ & \\
\hline HTN & & & & \\
\hline Yes & $298(68.8)$ & $122(40.9)$ & $176(59.1)$ & $<0.001$ \\
\hline No & $135(31.2)$ & $14(10.4)$ & $121(89.6)$ & \\
\hline IDDM & & & & \\
\hline Yes & $59(13.6)$ & $32(54.2)$ & $27(45.8)$ & $<0.001$ \\
\hline No & $374(86.4)$ & $104(27.8)$ & $270(72.2)$ & \\
\hline NIDDM & & & & \\
\hline Yes & $42(9.7)$ & $13(31)$ & $29(69)$ & 0.999 \\
\hline No & $391(90.3)$ & $123(31.5)$ & $268(68.5)$ & \\
\hline CAD/PVD/CVA & & & & \\
\hline Yes & $63(14.6)$ & $30(47.6)$ & $33(52.4)$ & 0.003 \\
\hline No & $369(85.4)$ & $105(28.5)$ & $264(71.5)$ & \\
\hline
\end{tabular}


Table 1. (continued)

\begin{tabular}{|c|c|c|c|c|}
\hline Characteristic & Overall $(n=434)$ & Dialysis $(n=136)$ & No dialysis $(\mathrm{n}=298)$ & $\mathrm{p}$ value \\
\hline \multicolumn{5}{|l|}{ AI disease } \\
\hline Yes & $127(29.4)$ & $30(23.6)$ & $97(76.4)$ & 0.030 \\
\hline \multicolumn{5}{|c|}{ Proteinuria at time of biopsy, $g$} \\
\hline Mean \pm SD & $3.9 \pm 4.4$ & $5.2 \pm 4.4$ & $3.3 \pm 4.2$ & $<0.001$ \\
\hline Median and range & $2.4(0-34.4)$ & $4.2(0-20.5)$ & $1.9(0-34.4)$ & \\
\hline Yes & $174(40.1)$ & $81(46.6)$ & $93(53.4)$ & $<0.001$ \\
\hline No & $260(59.9)$ & $55(21.2)$ & $205(78.8)$ & \\
\hline
\end{tabular}

Data are presented as mean $\pm \mathrm{SD}$, median (range), and $\mathrm{n}(\%)$.

$\mathrm{p}<0.05$ was considered significant.

IgA = Immunoglobulin A; MDRD = Modification of Diet in Renal Disease; NIDDM = non-insulin dependent DM; CVA = cerebrovascular accident.

\section{Results}

\section{Patient Characteristics and Risk Factors for Dialysis}

We identified 434 native kidney biopsies done at BUMC between 2001 and 2012. Baseline patient characteristics are summarized in table 1 . Over a median follow-up of $5.6(0.46-11.82)$ years, $136(31 \%)$ patients reached dialysis. Median time to dialysis was $0.86(-0.03$ to 10.6) years, including 6 patients who had dialysis immediately preceding the biopsy. During follow-up 15 (3.5\%) patients died and median time to death was 2.18 years $(0-10.4)$. The mean age at the time of biopsy was $45 \pm 16$. In our cohort, $42 \%$ of the patients were black, $22 \%$ were white, and $22 \%$ were Hispanic. The biopsies were performed in patients with various etiologies of chronic renal failure and a wide range of renal functions (creatinine $2.6 \pm 2.6 \mathrm{mg} / \mathrm{dl}$ and GFR $57 \pm 46 \mathrm{ml} / \mathrm{min}$ ). The mean fibrosis at the time of biopsy was $31.2 \pm 25.0 \%$ and mean glomerulosclerosis was $25.0 \pm 24.5 \%$. Patients who reached dialysis during the follow-up period were older ( $49 \pm 16$ vs. $43 \pm 16$ years) and dialysis was more likely to occur in male patients ( 38 vs. $24 \%$ ). We noted a significant racial difference with the highest rate of dialysis in blacks (39\%) and lower rates in whites (27\%) and Hispanics (25\%). Other clinical characteristics at baseline that were associated with a higher incidence of dialysis were HTN (41 vs. 10\%), insulin-requiring DM (54 vs. $28 \%$ ), cardiovascular disease ( 48 vs. $29 \%$ ), higher levels of proteinuria ( $5.2 \pm 4.4$ vs. $3.3 \pm 4.2 \mathrm{~g}$ ), especially nephrotic range proteinuria ( 47 vs. $21 \%$ ), higher creatinine $(4.3 \pm 3.2$ vs. $1.8 \pm 1.9 \mathrm{mg} / \mathrm{dl})$, and lower GFR $(29.4 \pm 28.9$ vs. $70.5 \pm 46.6 \mathrm{ml} / \mathrm{min}$ ). A history of AI disease carried a
Table 2. Number and percentage of patients with various degree of interstitial fibrosis, stratified by GFR

\begin{tabular}{llllr}
\hline GFR & $\begin{array}{l}\text { Interstitial } \\
\text { fibrosis } \\
<25 \%, \mathrm{n}(\%)\end{array}$ & $\begin{array}{l}\text { Interstitial } \\
\text { fibrosis } \\
25-49 \%, \mathrm{n}(\%)\end{array}$ & $\begin{array}{l}\text { Interstitial } \\
\text { fibrosis } \\
>50 \%, \mathrm{n}(\%)\end{array}$ & Total \\
\hline $60+$ & $139(80.81)$ & $24(13.95)$ & $9(5.23)$ & 172 \\
$30-59$ & $29(27.62)$ & $52(49.52)$ & $24(22.86)$ & 105 \\
$15-29$ & $12(14.12)$ & $28(32.94)$ & $45(52.94)$ & 85 \\
$<15$ & $19(26.39)$ & $13(18.06)$ & $40(55.56)$ & 72 \\
Total & 199 & 117 & 118 & 434 \\
\hline
\end{tabular}

lower risk of dialysis ( 23.6 vs. $34.4 \%$ ). As expected, more interstitial fibrosis $(51.6 \pm 23.7$ vs. $21.9 \pm 19.4 \%)$ and glomerular sclerosis $(40.1 \pm 28.4$ vs. $18.1 \pm 18.8 \%)$ were associated with dialysis. Patients with extensive interstitial fibrosis on biopsy but normal renal function had a median glomerulosclerosis score of 30 (range 5.7-66.7) that was similar to patients who had CKD. In unadjusted analysis, FGG was significantly associated with progression to dialysis $(\mathrm{p}<0.001)$ but when adjusting for other factors FGG lost significance. Being on insulin treatment was a risk factor for dialysis, but being on oral agents was not.

The majority of the 172 patients who had a GFR $>60$ $\mathrm{ml} / \mathrm{min}$ had less than $25 \%$ fibrosis on biopsy ( 139 or $81 \%$ ), but surprisingly, $5 \%$ of these patients with near normal renal function had more than $50 \%$ fibrosis and $13.9 \%$ had $25-49 \%$ fibrosis (table 2; fig. 1). As expected [16], with decreasing GFR, there were more patients in the $>50 \%$ 


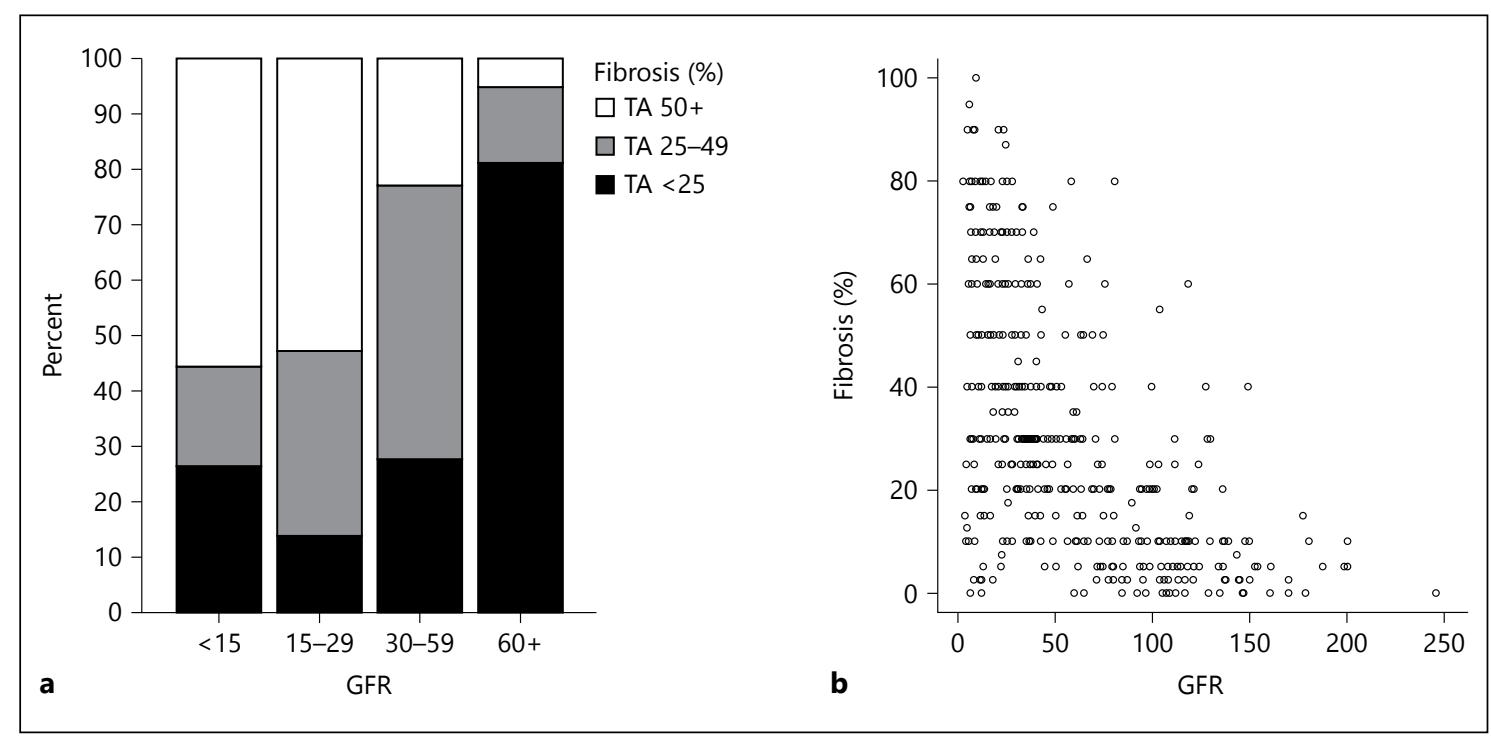

Fig. 1. Correlation between GFR and degree of fibrosis. a Percentage of fibrosis according to GFR levels. b A scatter plot of the overall cohort mapping the association of GFR with percent fibrosis. Each dot represents a single patient.

fibrosis group. Individual patient-level data on association between percentage of fibrosis and GFR are shown in figure $1 b$.

\section{Prediction of Dialysis}

We designed a prediction model using demographic, clinical, and biopsy variables collected at the time of biopsy to predict progression to dialysis (fig. 2a, b; online suppl. table 1a, b). The model included the following 6 factors: percentage of fibrosis, BMI, GFR, proteinuria, and diagnoses of HTN and IDDM (fig. 2a). The model has very good discriminative ability $(\mathrm{C}=0.879)$ and fit (Hosmer-Lemeshow $\mathrm{p}=0.565$ ). Interestingly, only GFR below $30 \mathrm{ml} / \mathrm{min}$ was predictive of future dialysis (online suppl. table $2 \mathrm{a}-\mathrm{c}$ ). The fibrosis alone model also had good characteristics with slightly lower discriminatory ability $(\mathrm{C}=0.832$, Hosmer-Lemeshow $\mathrm{p}=0.174$; fig. 2b). However, the 6-factor model was statistically superior (likelihood ratio test $\mathrm{p}<0.001)$ to the fibrosis-alone model. ROCs for both models are shown in figure $2 \mathrm{a}$ and $\mathrm{b}$.

\section{Interstitial Fibrosis and Dialysis in Different Patient Subgroups}

We examined the association between the level of fibrosis and dialysis status in different clinical subgroups. It is critical to note that in all groups, the range of fibrosis was wide. While fibrosis was a powerful predictor of dialysis risk overall (table 3), it was not predictive for some individuals. Regardless of the patients' diagnosis and demographics, there was a wide range of fibrosis observed in both the dialysis and non-dialysis groups. Some patients with $80 \%$ fibrosis on biopsy did not progress to ESRD (table 3; 'no dialysis' range of fibrosis $=0-80 \%$, table 1 ; interstitial fibrosis $0-80 \%$ in the 'no dialysis' group). There was an age-associated increase in the level of fibrosis in the patients who did not progress to dialysis (table 3; $14.8<19.1<29.5<31.5 \%)$ indicating that moderate levels of fibrosis did develop with aging and this was not necessarily indicative of dialysis risk. While in all 3 younger groups who progressed to ESRD the mean level of fibrosis was similar (51-53.1\%), the mean percent of fibrosis of $43.3 \%$ was lower in older patients $(>70)$ who progressed to dialysis. There were large statistically significant differences in fibrosis level between dialysis and non-dialysis patients for the 3 younger age groups $(\mathrm{p}<0.001)$. However, there was a much smaller difference in fibrosis level in the older patients and it did not reach statistical significance $(\mathrm{p}=0.148)$. The difference between the dialysis versus non-dialysis groups of patients with diabetic nephropathy on biopsy also did not reach statistical significance (54.2 vs. $43.5 \%, \mathrm{p}=0.167)$.

\section{Predictive Value of Interstitial Fibrosis}

We examined the value of interstitial fibrosis as a sole predictor of dialysis in different patient subgroups (online suppl. table 3). In most groups, fibrosis was a strong 


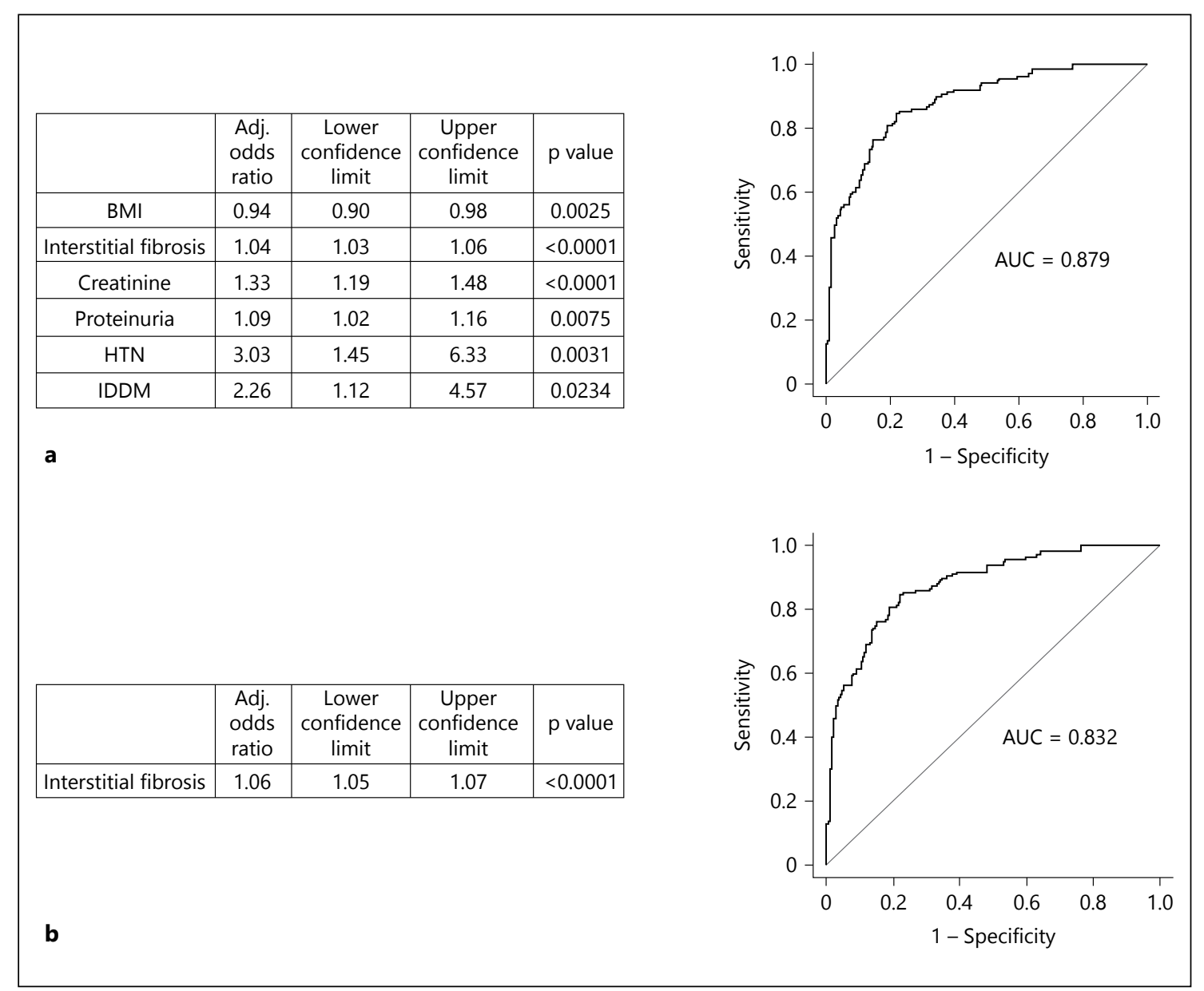

Fig. 2. Dialysis prediction model. Parsimonious prediction model for reaching dialysis. See Methods for a detailed description of the prediction model development. a Dialysis prediction model using 6 variables: parsimonious 0.2 level (left panel) and ROC for 6 variable model predicting dialysis (right panel) (variables included:

predictor of progression. However, we found 3 subgroups of patients in which fibrosis did not predict progression. Fibrosis was better as a predictor of dialysis in the younger age groups, but with increasing age, the association became less strong. In older patients ( $>70$ years), who represented $7.1 \%$ of our kidney biopsy cohort, fibrosis was not a statistically significant predictor of dialysis. Similarly, fibrosis was not a statistically significant predictor of dialysis in patients with diabetic nephropathy on biopsy or those receiving insulin therapy. In contrast, analysis of patients younger than 70 years who were non-diabetic showed that fibrosis is a good predictor of progression to ESRD both in our full-prediction model $(\mathrm{C}=$ $0.912)$ and in our fibrosis-only model $(\mathrm{C}=0.866$; online suppl. table 4).
BMI, percentage of interstitial fibrosis, creatinine, proteinuria, HTN, IDDM). AUC = Area under the curve. $\mathbf{b}$ Interstitial fibrosis alone dialysis prediction model (left panel) and ROC for dialysis prediction model using interstitial fibrosis only.

Percentage of Interstitial Fibrosis and Time to Dialysis

We tested fibrosis as a predictor of time to dialysis (table $4 a$; fig. 3,4$)$. Patients were categorized by the level of fibrosis into 3 groups with $0-24(\mathrm{n}=199), 25-49(\mathrm{n}=$ $117)$, or $>50(n=118)$ percent. As expected, the group with $>50 \%$ fibrosis progressed to ESRD more rapidly that those with either $25-49$ or $0-24 \%$ fibrosis with a median time to dialysis of 1.2 years compared to 6.5 and $>10$ years, respectively ( $\mathrm{p}<0.001$; table 4 ; fig. 4$)$. Note that 2 and 5 years after undergoing biopsy 42 and $21 \%$ of patients with $>50 \%$ fibrosis still remained dialysis free (table 4a; fig. 4). Cox regression analysis showed that having $>50$ or $25-49 \%$ fibrosis significantly increased the risk of dialysis with HRs of 7.2 (95\% CI 3.5-14.8) and 2.7 (95\% CI 1.3-5.3), respectively, compared to less than $25 \%$ fi- 
Table 3. Analysis of percentage fibrosis between dialysis group and no dialysis group for each patient characteristic

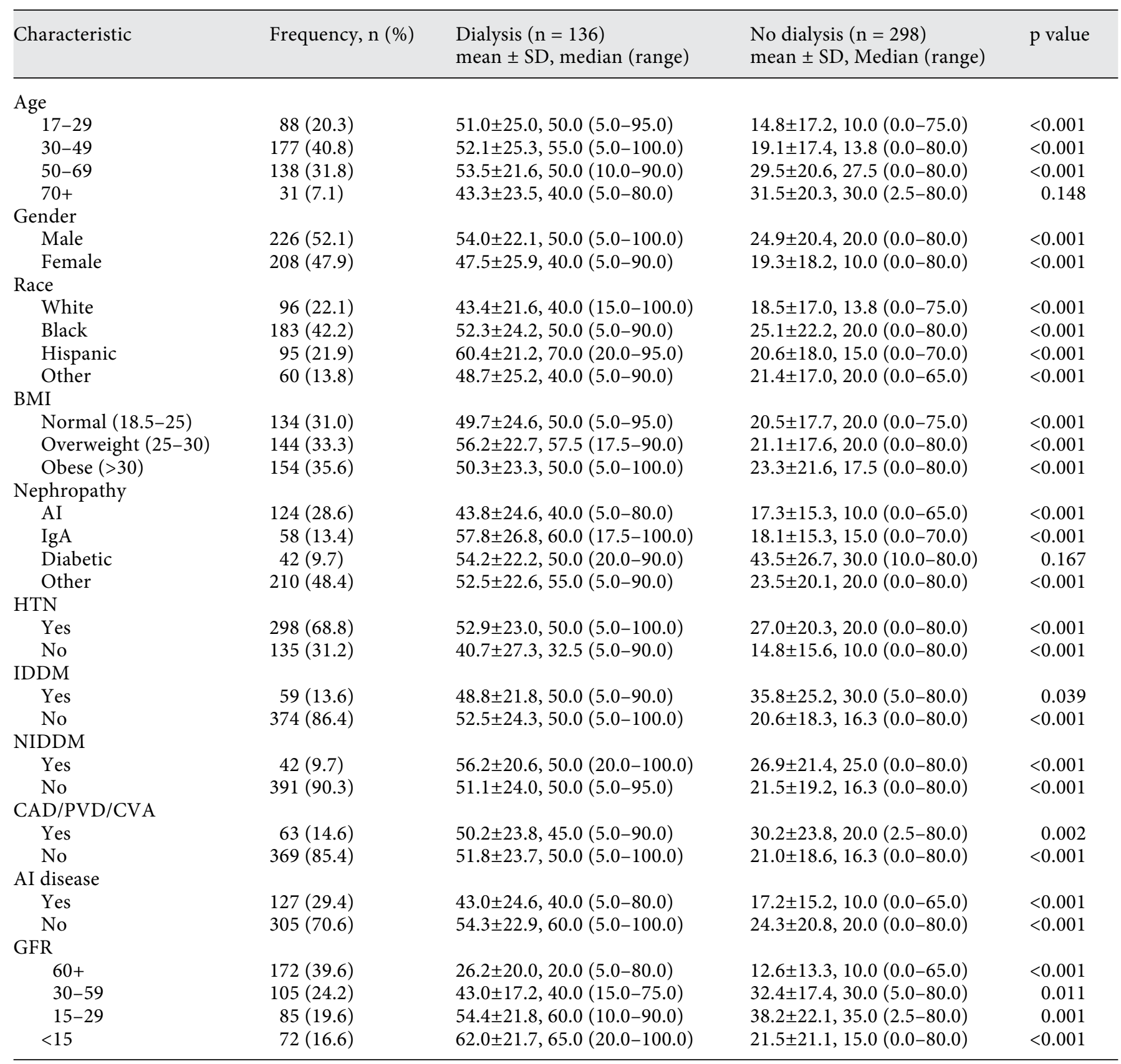

IgA = Immunoglobulin A; NIDDM = non-insulin dependent diabetes mellitus; CVA = cerebrovascular accident. $\mathrm{p}<0.05$ was considered significant.

brosis (table $4 \mathrm{~b}$ ). Lower BMI, GFR $<30$, and proteinuria $>3.5 \mathrm{~g}$ were the best predictors of progression to dialysis along with percent fibrosis. We found similar results using dialysis or death as end point instead of dialysis (online suppl. table $5 \mathrm{a}, \mathrm{b}$ and fig. 1 ).

\section{Discussion}

Our study highlights some of the limitations of using the level of interstitial fibrosis on renal biopsy to predict who will develop ESRD. Several studies have shown that 


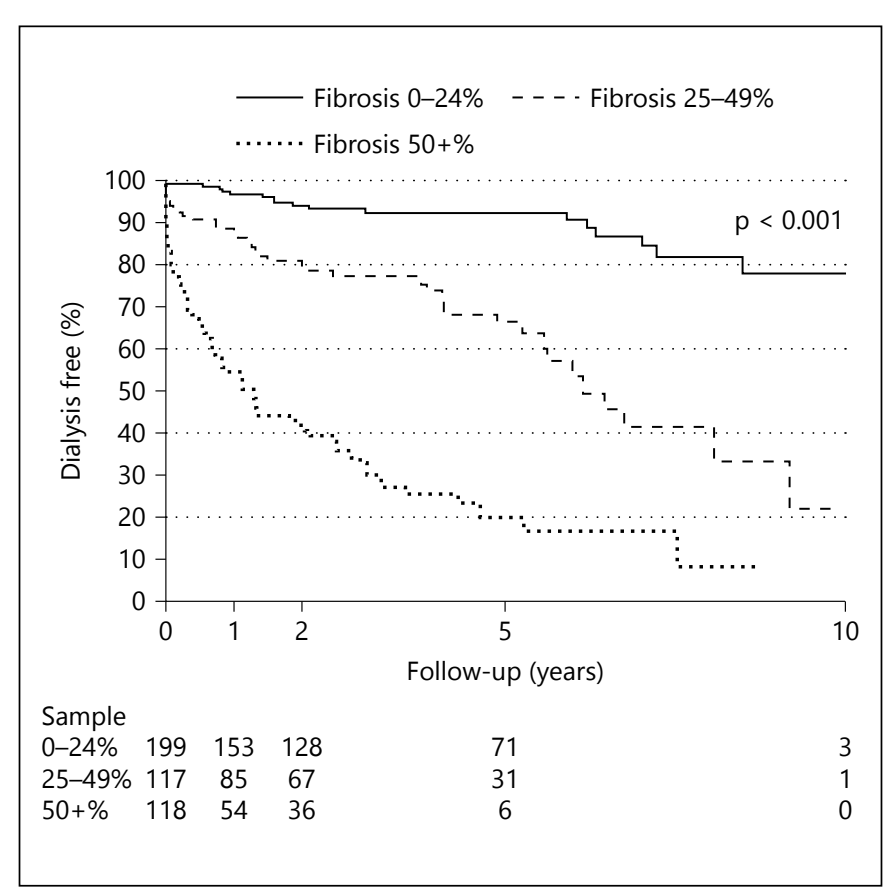

Fig. 3. Kaplan-Meier analysis of time-to-dialysis according to the level of interstitial fibrosis.

fibrosis on biopsy is an index of functional renal impairment and a major determinant in the progression of CKD $[1,5,6,10-12,17]$. Our findings emphasize that although fibrosis performs well overall, it is of less value for a subset of individual cases. Regardless of patient characteristics, we observed a wide range of fibrosis both in patients who reached dialysis and who remained dialysis free. Surprisingly, some of the patients who had $>80 \%$ fibrosis on the biopsy did not progress to ESRD during a 10-year followup period. Overreliance on fibrosis on biopsy may therefore erroneously identify patients as having advanced CKD at a time when interventions may still be effective.

Extent of fibrosis is a determining factor in designing clinical trials and in kidney transplantation. Patients with extensive histologic evidence of renal scarring with the percentage of fibrosis exceeding $50 \%$ are excluded from clinical trials because they are considered poor candidates for the evaluation of the effect of the therapy. In particular, they are routinely excluded from trials using medications that are designed to modulate immune response and functionally important inflammatory nephritis [18]. However, our data demonstrate that 42 and $21 \%$ of these patients are dialysis free at years 2 and 5, respectively, suggesting that some of those patients might also benefit from treatment. Patients who would benefit from a kidney transplant may

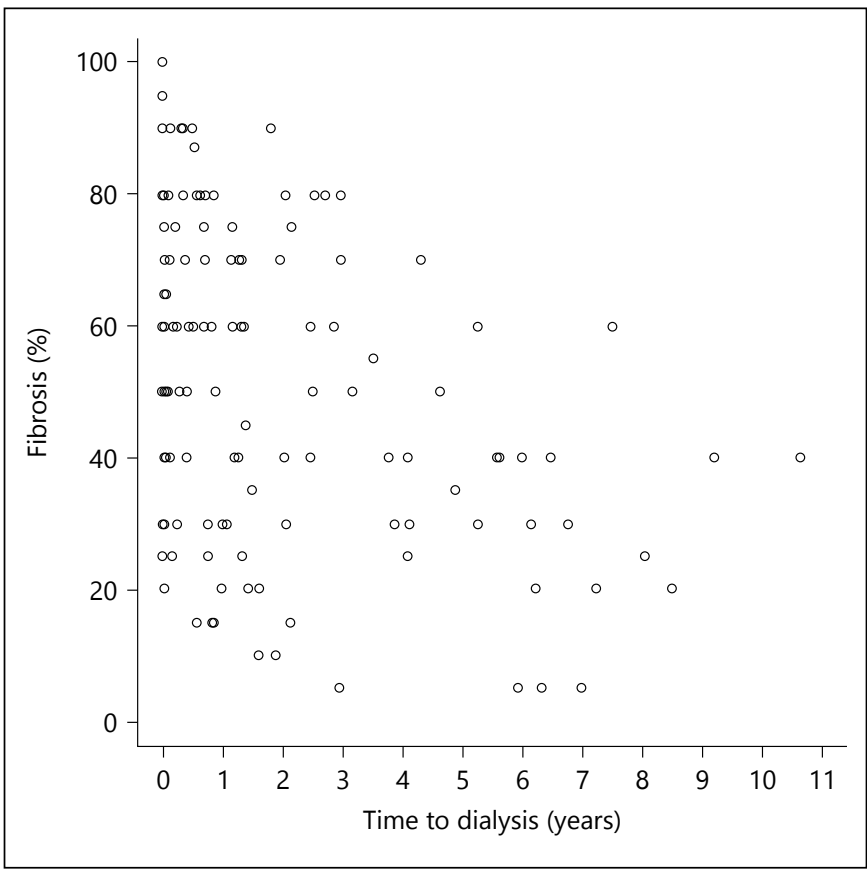

Fig. 4. Correlation between fibrosis and time to dialysis. A scatter plot of the overall cohort mapping the association of percentage fibrosis and time to dialysis. Each dot represents a single patient.

have to remain on the waitlist because kidneys with mild interstitial fibrosis are being rejected for transplantation [19]. Our study conducted in native kidneys is consistent with several studies in the transplant literature showing that a mild-to-moderate degree of fibrosis on time-zero biopsy does not necessarily predict worse graft function in living or deceased donor kidney transplantation [20-23].

To the best of our knowledge, this is the first study to identify specific patient groups where fibrosis does not perform well in predicting progression to ESRD: (1) in patients older than 70 years of age or (2) patients with diabetic nephropathy on biopsy. Our data show that aging leads to an increase in the level of fibrosis even in patients who do not progress to ESRD, making fibrosis a less reliable prognostic factor in the elderly. Age-associated glomerulosclerosis may in part explain the weaker correlation in the elderly patients. A previous study from 1991 with a large cohort of 488 patients with diabetic nephropathy had suggested that fibrosis correlates with progression to ESRD [24]. In contrast, in our cohort of 42 patients with diabetic nephropathy on biopsy, the level of interstitial fibrosis was not a reliable predictor of progression. One possible explanation for the divergent finding is the changing nature of diagnosis and treatment of patients with diabetic nephropathy. Patients with poorly 
Table 4.

a Time to event (dialysis) analysis. Life table estimates of dialysis for 10-year follow-up from biopsy by severity of fibrosis at biopsy

\begin{tabular}{lccc}
\hline Had events up to: & \multicolumn{2}{l}{ Interstitial fibrosis $(95 \% \mathrm{CI})$} & \\
\cline { 2 - 4 } & $0-24 \%$ & $25-49 \%$ & $180 \%$ \\
\hline 1 month & $1.0(0.2-3.4)$ & $6.0(2.7-11.4)$ & $33.8(25.3-42.5)$ \\
6 months & $1.0(0.2-3.4)$ & $9.6(5.1-15.9)$ & $45.4(35.9-54.4)$ \\
1 year & $3.4(1.4-6.9)$ & $11.6(6.5-18.3)$ & $58.3(48.1-67.1)$ \\
years & $6.1(3.1-10.5)$ & $19.4(12.4-27.6)$ & $79.1(68.2-86.6)$ \\
10 years & $8.0(4.3-13.2)$ & $33.0(23.0-43.3)$ & $89.6(71.0-96.5)$ \\
\hline
\end{tabular}

b Dialysis risk factor analysis. Cox proportional hazard model for time to dialysis

\begin{tabular}{|c|c|c|c|c|}
\hline & Adjusted HR & $\begin{array}{l}\text { Lower confidence } \\
\text { limit }\end{array}$ & $\begin{array}{l}\text { Upper confidence } \\
\text { limit }\end{array}$ & $\mathrm{p}$ value \\
\hline \multicolumn{5}{|l|}{ TA } \\
\hline $25-49$ vs. $0-24$ & 2.7 & 1.3 & 5.3 & 0.003 \\
\hline $50+$ vs. $0-24$ & 7.2 & 3.5 & 14.8 & $<0.001$ \\
\hline Age & 1.0 & 0.9 & 1.0 & 0.587 \\
\hline Male vs. female & 1.2 & 0.8 & 1.8 & 0.361 \\
\hline White vs. black & 1.5 & 0.8 & 2.6 & 0.147 \\
\hline Hispanic vs. black & 0.9 & 0.5 & 1.6 & 0.895 \\
\hline BMI & 0.9 & 0.9 & 0.9 & 0.004 \\
\hline FGG & 1.0 & 0.9 & 1.0 & 0.373 \\
\hline \multicolumn{5}{|l|}{ GFR } \\
\hline $30-59$ vs. $60+$ & 0.9 & 0.4 & 1.9 & 0.950 \\
\hline $15-29$ vs. $60+$ & 2.5 & 1.3 & 4.8 & 0.004 \\
\hline$<15$ vs. $60+$ & 8.3 & 4.3 & 15.8 & $<0.001$ \\
\hline Protein: $3.5+$ vs. $<3.5$ & 2.0 & 1.4 & 3.0 & $<0.001$ \\
\hline $\mathrm{HTN}$ & 1.8 & 0.9 & 3.4 & 0.053 \\
\hline CAD & 1.1 & 0.6 & 1.8 & 0.643 \\
\hline \multicolumn{5}{|l|}{ Nephropathy } \\
\hline Diabetic vs. AI & 0.7 & 0.3 & 1.54 & 0.482 \\
\hline IgA vs. AI & 0.7 & 0.3 & 1.7 & 0.526 \\
\hline Other vs. AI & 0.6 & 0.3 & 1.1 & 0.118 \\
\hline
\end{tabular}

controlled diabetes and other obvious microvascular complications are frequently not biopsied. As a result, we have a lower biopsy rate in these patients compared to other etiologies and this potentially could lead to sampling bias. Alternatively, the poor correlation between fibrosis and ESRD progression in our study could be because diabetic patients usually have some level of chronic vascular injury that can be patchy leading to variable levels of fibrosis in the tissue.

Another helpful clinical tool, in conjunction with predicting if a patient will progress to dialysis, is anticipating the amount of time a patient has until dialysis initiation (table 4a; fig. 3, 4). This data could be used in clinical practice to give a rough estimate of dialysis-free survival to our patients based on their level of fibrosis. In general, the group with more fibrosis progressed to ESRD more rapidly than those with less fibrosis, but again, individual variability was large regardless of biopsy diagnosis or patient characteristics.

We noted several expected findings that confirmed previous observations. Patients who reached dialysis during the follow-up period were older and more likely to be male. HTN, nephrotic range proteinuria, vascular disease, and worse renal function at the time of biopsy were all risk factors for dialysis. Being on insulin treatment was a risk factor for dialysis, but being on oral agents was not a risk factor, suggesting that easily controlled diabetics often do not progress to ESRD. History of AI diseases car- 
ried a lower risk of dialysis likely because these groups of pathologies are potentially treatable.

All biopsies were read by one of our 2 pathologists; therefore, the pathological findings are internally consistent. In general, the reliability of pathologist-based estimates of fibrosis improves the likelihood that our findings can be accurately reproduced in other centers. Furthermore, because interstitial fibrosis is one of the common final pathways in all kidney diseases, the results of this study are widely applicable.

Another important strength of our study is that its conclusions carry across racial lines. Our patient population was diverse: $42 \%$ of the patients were black, $22 \%$ were white, and 22\% were Hispanic. Renal disease disproportionately affects African-Americans; therefore, it is imperative to validate study findings in this population. Similar to previous studies we found that blacks were more likely to progress to ESRD regardless of their diagnosis.

Death rate was relatively low in our cohort. Only 15 (3.46\%) patients died during the study period. The median time to death was 2.18 years. We speculate that very sick patients who died soon after presentation were not biopsied. The long duration of follow-up (median 5.6 years), large sample size, low rate of loss to follow-up, and availability of detailed medical history in electronic medical records are additional strengths.

In our cohort, a large proportion of renal biopsies were done at CKD stages 3-4 with mean creatinine of $2.6 \pm 2.6$ $\mathrm{mg} / \mathrm{dl}$. Most of these patients likely had renal impairment, proteinuria, or hematuria for a prolonged period of time before they were referred to a nephrologist and a biopsy was done.

The retrospective nature of this study is a limitation. Another potential limitation is that timing of the biopsy and dialysis initiation can be physician dependent and could contribute to lead time bias. In our opinion, in a university teaching hospital setting, nephrologists follow the same guidelines, so it is unlikely that provider variability had a large effect on renal outcome or timing of dialysis. Results for specific subgroups of patients were based on limited sample sizes and should be confirmed in a larger cohort. Last, our study results were derived from a single cohort and will require validation in an external cohort. While the inclusion of additional information in a predictive model resulted in statistically significant improvement in discriminatory ability, it is not clear whether this improvement is clinically relevant. How multiple predictors could be used simultaneously in clinical practice also warrants further study.

Our study identifies some of the limitations in establishing disease prognosis and progression from a kidney biopsy obtained at one point in the course of a chronic disease affected by sampling error. Development of a noninvasive technique such as transient elastography for liver fibrosis [25-28] would be ideal for repeated, global assessment of renal fibrosis and to determine whether or not it correlates with functional deterioration. In the meantime, we should be cautious about the interpretation of renal fibrosis, especially in the elderly and those with diabetic nephropathy.

\section{Acknowledgments}

This research was partially supported by NIH grant 1KO8DK090143 (A.H.). We thank Prof. David J. Salant for his comments that greatly improved the manuscript.

\section{Disclosure Statement}

None.

\section{References}

1 Kostadinova-Kunovska S, Petrusevska G, Jovanovic R, Grcevska L, Bogdanovska M, Polenakovic M: Morphological changes in the tubulointerstitial compartment in primary glomerulopathies. Prilozi 2007;28:6174.

2 Madaio MP: Renal biopsy. Kidney Int 1990; 38:529-543.

3 Serón D, Moreso F, Fulladosa X, Hueso M, Carrera M, Grinyó JM: Reliability of chronic allograft nephropathy diagnosis in sequential protocol biopsies. Kidney Int 2002;61:727733.
4 Walsh M, Sar A, Lee D, Yilmaz S, Benediktsson H, Manns B, Hemmelgarn B: Histopathologic features aid in predicting risk for progression of IgA nephropathy. Clin J Am Soc Nephrol 2010;5:425-430.

5 Nath KA: Tubulointerstitial changes as a major determinant in the progression of renal damage. Am J Kidney Dis 1992;20:1-17.

6 Risdon RA, Sloper JC, De Wardener HE: Relationship between renal function and histological changes found in renal-biopsy specimens from patients with persistent glomerular nephritis. Lancet 1968;2:363-366.
7 Rodríguez-Iturbe B, Johnson RJ, HerreraAcosta J: Tubulointerstitial damage and progression of renal failure. Kidney Int Suppl 2005;99:S82-S86.

8 D'Amico G: Influence of clinical and histological features on actuarial renal survival in adult patients with idiopathic IgA nephropathy, membranous nephropathy, and membranoproliferative glomerulonephritis: survey of the recent literature. Am J Kidney Dis 1992;20:315-323.

9 Meyer TW: Tubular injury in glomerular disease. Kidney Int 2003;63:774-787. 
10 Nagy J, Trinn C, Deák G, Schmelzer M, Burger T: The role of the tubulointerstitial changes in the prognosis of IgA glomerulonephritis. Klin Wochenschr 1984;62:1094-1096.

11 Wang H, Tomino Y, Fukui M, Shirato I, Yaguchi Y, Ebihara I, Kubota M, Nakayama S, Koide H: Correlation between tubulointerstitial changes and prognosis in patients with primary membranous nephropathy. Nephron 1994;67:362.

12 Ziyadeh FN: Significance of tubulointerstitial changes in diabetic renal disease. Kidney Int Suppl 1996;54:S10-S13.

$13 \mathrm{http}: / / \mathrm{mdrd} . c o m /$.

14 Cerqueira DC, Soares CM, Silva VR, Magalhães JO, Barcelos IP, Duarte MG, Pinheiro SV, Colosimo EA, Simões e Silva AC, Oliveira EA: A predictive model of progression of CKD to ESRD in a predialysis pediatric interdisciplinary program. Clin J Am Soc Nephrol 2014;9:728-735.

15 Tangri N, Stevens LA, Griffith J, Tighiouart H, Djurdjev O, Naimark D, Levin A, Levey AS: A predictive model for progression of chronic kidney disease to kidney failure. JAMA 2011;305:1553-1559.

16 Mackensen-Haen S, Bader R, Grund KE, Bohle A: Correlations between renal cortical interstitial fibrosis, atrophy of the proximal tubules and impairment of the glomerular filtration rate. Clin Nephrol 1981;15:167-171.

17 Park MH, D’Agati V, Appel GB, Pirani CL: Tubulointerstitial disease in lupus nephritis: relationship to immune deposits, interstitial inflammation, glomerular changes, renal function, and prognosis. Nephron 1986;44: 309-319.

18 Ballardie FW, Roberts IS: Controlled prospective trial of prednisolone and cytotoxics in progressive IgA nephropathy. J Am Soc Nephrol 2002;13:142-148.

19 Snoeijs MG, Buurman WA, Christiaans MH, van Hooff JP, Goldschmeding R, van Suylen RJ, Peutz-Kootstra CJ, van Heurn LW: Histological assessment of preimplantation biopsies may improve selection of kidneys from old donors after cardiac death. Am J Transplant 2008;8:1844-1851.

20 Lee AL, Kim YS, Lim BJ, Jeong HJ, Joo DJ, Kim MS, Huh KH: The impact of time-zero biopsy on early graft outcomes after living donor kidney transplantation. Transplant Proc 2013;45:2937-2940.

21 Sund S, Reisaeter AV, Fauchald P, Bentdal O, Hall KS, Hovig T: Living donor kidney transplants: a biopsy study 1 year after transplantation, compared with baseline changes and correlation to kidney function at 1 and 3 years. Nephrol Dial Transplant 1999;14: 2445-2454.

22 Cockfield SM, Moore RB, Todd G, Solez K, Gourishankar S: The prognostic utility of deceased donor implantation biopsy in determining function and graft survival after kidney transplantation. Transplantation 2010; 89:559-566.

23 El-Husseini A, Sabry A, Zahran A, Shoker A: Can donor implantation renal biopsy predict long-term renal allograft outcome? Am J Nephrol 2007;27:144-151.

24 Bohle A, Wehrmann M, Bogenschütz O, Batz C, Müller CA, Müller GA: The pathogenesis of chronic renal failure in diabetic nephropathy. Investigation of 488 cases of diabetic glomerulosclerosis. Pathol Res Pract 1991;187: 251-259.

25 Macías J, Camacho A, Von Wichmann MA, López-Cortés LF, Ortega E, Tural C, Ríos MJ, Merino D, Téllez F, Márquez M, Mancebo M, Pineda JA: Liver stiffness measurement versus liver biopsy to predict survival and decompensations of cirrhosis among HIV/hepatitis $C$ virus-coinfected patients. AIDS 2013; 27:2541-2549.

26 Singh S, Fujii LL, Murad MH, Wang Z, Asrani SK, Ehman RL, Kamath PS, Talwalkar JA: Liver stiffness is associated with risk of decompensation, liver cancer, and death in patients with chronic liver diseases: a systematic review and meta-analysis. Clin Gastroenterol Hepatol 2013;11:1573-1584; quiz e88-e89.

27 Awad Mel-D, Shiha GE, Sallam FA, Mohamed A, El Tawab A: Evaluation of liver stiffness measurement by fibroscan as compared to liver biopsy for assessment of hepatic fibrosis in children with chronic hepatitis $\mathrm{C}$. J Egypt Soc Parasitol 2013;43:805-819.

28 Kim BK, Fung J, Yuen MF, Kim SU: Clinical application of liver stiffness measurement using transient elastography in chronic liver disease from longitudinal perspectives. World J Gastroenterol 2013;19:1890-1900. 